\title{
ENDURANCE LIMIT OF THE AXIAL-PISTON HYDRAULIC MACHINE CYLINDER BLOCK
}

\author{
Yuriy KHOMIAK ${ }^{1}$, Oleksandr KIBAKOV ${ }^{2}$, Stanislav MEDVEDEV ${ }^{2}$, \\ Ilya NIKOLENKO ${ }^{3}$, Victoria ZHEGLOVA ${ }^{1}$ \\ ${ }^{1}$ Odessa National Polytechnic University, Ukraine \\ ${ }^{2}$ Odessa National Maritime University, Ukraine, e-mail:kibakov60@gmail.com \\ ${ }^{3}$ Academy of Construction and Architecture, "V.I. Vernadsky Crimean Federal University"
}

\begin{abstract}
The fatigue resistance of the cylinder block (CB) of an axial-piston hydraulic machine (APHM) made of tin bronze CuSn12 has been investigated. Fatigue tests of smooth and notched standard samples were performed and fatigue curves were constructed. The stress state of the $\mathrm{CB}$ is investigated by the finite element method. It is established that the maximum stresses are localized on a small part of the partition between axial cylinders holes loaded by internal pressure. A method for recalculating the established length of this zone of partition with an uneven distribution of dangerous stresses by an equivalent length with a constant stress equal to the maximum is proposed. The use of the statistical theory of the similarity of the fatigue failure of Serensen-Kogayev, the results of the fatigue tests of the samples and the calculation of the equivalent length made it possible to determine the endurance limit of the CB considered. The acceptability of the proposed method estimating the endurance limit by the known results of resource tests of the CB APHM 210.25 is confirmed.
\end{abstract}

Keywords: complex stress state; finite element method; fatigue fracture similarity criterion; fatigue curves of tin bronze CuSn12.

\section{INTRODUCTION}

The main trends in the development of the volumetric hydraulic drive are an increase in nominal pressure from $20 . .25 \mathrm{MPa}$ to $32 . .45 \mathrm{MPa}$ and a reduction in overall dimensions while maintaining the requirements for its reliability. The solution of these problems is obtained by improving the designs, increasing the quality of the materials of the parts, and the obligatory observance of maintenance requirements. The most important indicator of reliability is the durability of machines. The increase in pressure in axial piston hydraulic machines (APHM) leads to an increase in volumetric and contact stresses and a magnification in the frequency of failures, of which approximately $30 \%$ account for only two parts, such as the cylinder block (CB) and the distributor $[1,2]$. These failures are of two types: wear of contact surfaces and fatigue destruction. The reduction of wear in APHM is achieved by providing lubrication of contact surfaces with working fluid (mineral oil) and using up materials of details with high antifriction properties [3, 4]. It should also be taken into account that when the nominal pressure increases, the clearances in some connection increase. This leads to an increase in leakage and a decrease in the volumetric efficiency of the APHM $[5,6]$. Because of the large areas of the friction surfaces, to minimize wear, CB from a tin bronzes are produced. At high levels of cyclically changing pressure, the relatively low strength of bronze is the cause of $\mathrm{CB}$ fatigue destruction. The complexity of the multiply connected $\mathrm{CB}$ design does not allow to determine its stress-strain state and to establish the fatigue resistance characteristics by traditional analytical methods.

This article proposes a numerical-analytical method for calculating of CB APHM for fatigue resistance, which allowed find its endurance limit. On the basis of this method, it is possible to construct a fatigue curve for $\mathrm{CB}$, and the resource may be evaluated at given loading regimes.

\section{ANALYSIS OF THE LITERATURE DATA AND STATEMENT OF THE PROBLEM}

Stresses that arise in unevenly loaded details of complex shape are often localized in small internal volumes and it is only experimentally possible to effectively determine their effect on strength and fatigue resistance. Such works were carried out for local sections of notched samples of material [7], individual structure units $[8,9]$ and vehicles $[10$, 11] and even for multi-storey buildings [12] on special stands. Making stands and performing experiments are requires considerable expenditure of resources and time, therefore, in modern conditions, the analysis of strength and fatigue resistance is carried out numerically more often.

For the details by the finite element method $(\mathrm{FEM})$ in a three-dimensional formulation are 
determine all components of the stress tensor. The characteristics of strength and fatigue resistance are established for samples of the detail material on standard stands experimentally. Then, analysis of strength or fatigue resistance of a given detail is performed using approved calculation algorithms [13-14]. For details of simple shapes (rods, some plates), the methodology for calculating fatigue resistance in the current regulatory documents is proposed $[15,16]$. These documents cannot cover a wide range of details complex shape, including $\mathrm{CB}$, so special methods to calculate them are used [1720]. The CB APHM to a complex details class is related. The main load for the $\mathrm{CB}$ is the internal pressure $p$ acting on the parts of the axial cylindrical channels. The length of these parts is determined by the position of the pistons moving in the axial direction $z$. This type of loading leads to the appearance in the $\mathrm{CB}$ of a cyclically varying uneven stresses. The maximum values of these stresses in the short region of the inter-cylinder partition (ICP) are localized.

The aim of this paper is to create a method for calculating the fatigue resistance of details with the working stress localization, which is applied to CB APHM. The basis of the method is the statistical theory of the similarity of fatigue failure (STSFF) of Serensen-Kogayev [22], on which GOST 25.504-82 [16] is founded.

To achieve this purpose in the work of the following tasks:

- to build a fatigue curve and establish the endurance limit of the material $\mathrm{CB}$, tin bronze CuSn12;

- to determine the distribution and the stresses magnitude arising in the $\mathrm{CB}$ at the most unfavorable pressure propagation in its axial cylinders by the FEM;

- to demonstrate the procedure of calculating the fatigue resistance for details with a significantly uneven distribution of stresses on the example of determining the endurance limit of a CB APHM.

\section{List of symbols}

APHM Axial-piston hydraulic machine

CB Cylinder block

FEM Finite elements method

ICP Inter-cylinder partition

STSFF Statistical theory of the similarity of fatigue failure

C Parameter of fatigue curve equation

$d$ Diameter of the sample for fatigue tests

$G \quad$ Gradient of the first principal stress

$\bar{G}, \bar{G}_{0} \quad$ Relative gradient of the first principal stress of the detail and of the sample

$K_{A} \quad$ Anisotropy material factor

$K_{F} \quad$ Fatigue strength surface condition factor

$K_{t} \quad$ Theoretical stress concentration factor

$K_{V} \quad$ Fatigue surface hardening factor
$L \quad$ Length of the perimeter of crosssection or its part where the maximum stresses arise

$L_{0} \quad$ Length of the cross-section perimeter of the standard sample with a diameter of $7.5 \mathrm{~mm}$

$L_{1 e} \quad$ Equivalent length of the part of the perimeter of the cross-section of the detail within which the stresses are assumed to be equal to $\sigma_{\max }$

$L_{e} \quad$ Sum of all equivalent lengths $L_{1 e}$ of the detail

$m \quad$ Coefficient of slope fatigue curve constructed in double logarithmic coordinates

$N \quad$ Quantity of loading cycles before failure

$N_{B} \quad$ Test base

$N_{G} \quad$ Turning point of Wöhler curve

$p \quad$ Pressure of working fluid

$r \quad$ Correlation coefficient between stress values and life to failure

$R_{a} \quad$ Arithmetic average roughness height of surface

$R_{z} \quad$ Height roughness of surface by ten points

$S_{y} \quad$ Mean square deviation of a random variable $y_{i}=\log \left(\sigma_{\max }-u\right)$

$u \quad$ Lower limit of stresses at which fatigue damages are accumulated

$x, z \quad$ Circumference and axial coordinates

$t_{0} \quad$ Thickness of the ICP

$\delta \quad$ Elongation at break

$\theta$ Relative similarity criterion of fatigue failure

$v_{\sigma} \quad$ Coefficient of sensitivity to stress concentration and to influences of the size of details

$\xi \quad$ Relative maximum stress

$\sigma_{0.2} \quad$ Conventional yield strength

$\sigma_{\max } \quad$ Maximum value of first principal stress

$\sigma_{-1} \quad$ Endurance limit of the sample

$\sigma_{-1 D} \quad$ Endurance limit of material at symmetrical stress cycle

$\sigma_{-1 e} \quad$ Average value endurance limit of a detail at symmetrical cycle

$\sigma_{0 D} \quad$ Endurance limit under pulsation

$\sigma_{u} \quad$ Ultimate strength

$\omega, \sigma_{W} \quad$ Weibull's distribution parameters

\section{THE BASIS DESIGN DEPENDENCE}

The median value of the endurance limit of details is determined by the expression

$$
\bar{\sigma}_{-1 D}=\sigma_{-1} K_{V} K_{A} /\left[2 K_{t} /\left(1+\theta^{-v_{\sigma}}\right)+1 / K_{F}-1\right]
$$

The standard contains recommendations for estimating the parameters of equation (1): a median 
value of the endurance limit of the sample $\sigma_{-1}$ and the characteristics of the detail $K_{t}, v_{\sigma}, K_{V}, K_{A}, K_{F}$ [16]. The relative similarity criterion of fatigue failure is a ratio

$$
\theta=(L / \bar{G}) /\left(L_{0} / \bar{G}_{0}\right)
$$

where $\left(L_{0} / \bar{G}_{0}\right)=88.3 \mathrm{~mm}^{2}-$ the criterion for the similarity of smooth laboratory samples with a diameter of $7.5 \mathrm{~mm} ;(L / \bar{G})-$ the criterion for the similarity of the given detail; $\bar{G}_{0}$ and $\bar{G}-$ the relative gradients of the first principal stress in the zone of their concentration for the samples and the detail, respectively.

The parameter of the detail $L$ means the length of the perimeter of the dangerous section or the part of it where the maximum stresses act.

The main advantage of STSFF is the assertion that samples and details with different sizes and shapes, but with the same criterion values $(L / \bar{G})$ have equal endurance limits. Consequently, the limit of endurance of real details can be determined from the results of tests of laboratory samples.

\section{FATIGUE TESTS OF SAMPLES OF CB MATERIAL}

To determine the characteristics of the fatigue resistance of bronze $\mathrm{CuSn} 12$, from which the $\mathrm{CB}$ is made, fatigue tests of corset (smooth) and notched samples were performed (Fig. 1).

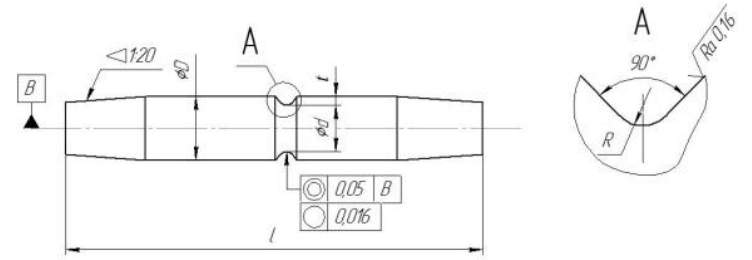

Fig. 1. The V-notched sample design

The main components of bronze: $\mathrm{Sn} 12 \%, \mathrm{~Pb}$ $1.3 \%$, $\mathrm{Ni} 1 \%$ (the rest is $\mathrm{Cu}$ ). Mechanical characteristics: ultimate strength $\sigma_{u}=320 \mathrm{MPa}$, yield strength $\sigma_{0.2}=180 \mathrm{MPa}$, elongation at break $\delta=12 \%$, hardness in the range of $56 \div 58 \mathrm{HRB}(101 \div 104 \mathrm{HB})$. The dimensions of the samples are given in Table 1 . Here is also presented the theoretical stress concentration factor $K_{t}(\mathrm{CSCF})$ for a cylindrical surface, which is determined by the Neuber formulas for the notch a hyperbolic profile [16]

$$
K_{t}=1+\frac{\left(K_{t 1}-1\right)\left(K_{t 2}-1\right)}{\sqrt{\left(K_{t 1}-1\right)^{2}+\left(K_{t 2}-1\right)^{2}}},
$$

where the theoretical stress concentration coefficients for shallow and deep notches are respectively equal

$$
\begin{gathered}
K_{t 1}=1+2 \sqrt{\frac{t}{\rho}} \\
K_{t 2}=\frac{3}{4} \cdot \frac{(1+\zeta)^{2}\left(3 \zeta^{2}-0.4 \zeta+1.3\right)}{3 \zeta^{3}+5.2 \zeta^{2}+2.2 \zeta+1.3}
\end{gathered}
$$

where $\zeta=\sqrt{1+\frac{a}{\rho}} ; a=0.5 d-$ the radius of dangerous section; $t$ - the depth of the notching (Fig. 1).

The design radius of curvature is $\rho=1.05 R$ because a hyperbolic notch profile is replaced by an equivalent V-notch rounded profile [21]. Similarity

\begin{tabular}{|c|c|c|c|c|c|c|}
\hline \multirow{2}{*}{$\begin{array}{c}\text { The shape } \\
\text { of the } \\
\text { working } \\
\text { part of the } \\
\text { sample }\end{array}$} & \multicolumn{4}{|c|}{ Dimensions, mm } & \multirow{2}{*}{$K_{t}$} & \multirow{2}{*}{$\begin{array}{c}L / \bar{G} \\
\mathrm{~mm}^{2}\end{array}$} \\
\hline & $D$ & $d$ & $t$ & $R$ & & \\
\hline Corsetry 100 & 11 & 7.5 & 1.75 & 75 & 1.01 & 78.1 \\
\hline $\begin{array}{c}\text { V-notched } \\
90^{\circ}\end{array}$ & 11 & 7.5 & 1.75 & 1 & 1.74 & 9.67 \\
\hline
\end{tabular}
criterion of fatigue failure $L / \bar{G}$ is set according to [16].

Table 1. The parameters of samples for fatigue tests

To prepare the samples, $20 \times 20 \mathrm{~mm}$ blocks were used, which were obtained from the longitudinally cut billet for a CB with a diameter of $120 \mathrm{~mm}$, followed by turning and grinding to a surface finish $R a 0.4 \mu \mathrm{m}(R z 1.6 \mu \mathrm{m})$. The manufacturing process was kept constant for the whole batch.

The tests were carried out with a pure circular bending with a loading frequency of $50 \mathrm{~Hz}$. The test base was $N_{B}=10^{8}$ cycles. To describe the fatigue curve, the power equation

$$
\sigma^{m} N=\sigma_{-1}^{m} N_{G}=10^{C}=\text { const },
$$

where $\sigma$ and $N$ - the current value of the test stress and the corresponding durability before failure; $m$ and $C-$ the parameters of equation (6); $\sigma_{-1}-$ the endurance limit of the material for a symmetric stress change cycle; $N_{G}$ - the abscissa of the fracture point of the fatigue curve plotted in double logarithmic coordinates.

The fatigue curves for smooth samples (round symbols) and for notched samples (quadrate symbols) constructed in double logarithmic coordinates according to the results of fatigue tests is presented in Fig. 2.

Dark symbols correspond to broken samples; light ones correspond to samples that did not crumble until the base of test $10^{8}$ cycles. As a result of fatigue testing and correlation and regression analysis, the characteristics of eq. (6) are obtained, which are presented in Table 2. Limits of endurance are defined as stress levels at which three of the two samples did not collapse. 


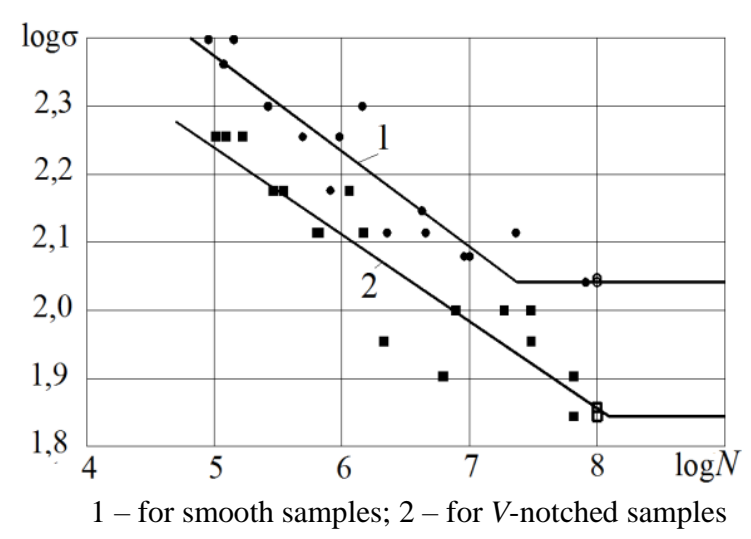

Fig. 2. The curves of fatigue

Table 2. The characteristics of the fatigue curve

\begin{tabular}{cccccc}
\hline $\begin{array}{c}\text { The shape } \\
\text { of the } \\
\text { working } \\
\text { part of the } \\
\text { sample }\end{array}$ & $m$ & $C$ & $\begin{array}{c}N_{G}, \\
\text { cycle }\end{array}$ & $\begin{array}{c}\sigma_{-1}, \\
\mathrm{MPa}\end{array}$ & $r$ \\
\hline $\begin{array}{c}\text { Corsetry } \\
\text { V-notched } \\
90^{\circ}\end{array}$ & 7.14 & 21.9 & $2.37 \cdot 10^{7}$ & 110 & -0.919 \\
\hline
\end{tabular}

The calculated large values of the modulus of correlation coefficients $r$ indicate that there is a strong correlation between $\log \sigma$ and $\log N$. Therefore, the use of equation (6) for the study bronze $\mathrm{CuSn} 12$ is quite justified.

To solve the problem considered in the article, it is necessary to determine the coefficient of sensitivity of the metal to stress concentration and the influence of absolute dimensions $v_{\sigma}$. For this we use the formula [22]

$$
S_{y}=0.62 v_{\sigma} /\left(1+0.36 v_{\sigma}\right)
$$

where $S_{y}=\sqrt{\frac{1}{n-1} \cdot\left[\sum_{i=1}^{n} y_{i}^{2}-\frac{1}{n} \cdot\left(\sum_{i=1}^{n} y_{i}\right)^{2}\right]}-$ the mean square deviation of a random variable $y_{i}=\log \left(\sigma_{\max }-u\right)$. The maximum stresses for each test level are found as $\sigma_{\max i}=K_{t} \cdot \sigma_{i}$, see Table 1 and Fig. 2. The lower boundary of stresses at which the fatigue damage is accumulated, for plastic materials (bronze CuSn12 has $\delta>10 \%$ therefore is plastic material) defined as $u=0,5 \cdot \sigma_{-1}=55 \mathrm{MPa}$. The calculations of the mean square deviation gave the following values: for smooth samples $S_{y 1}=0.175$, for notched samples $S_{y 2}=0.178$. From formula (7) the expression is obtained $v_{\sigma}=S_{y} /\left(0,62-0.36 S_{y}\right)$. Based on the calculated values $S_{y 1}$ and $S_{y 2}$ average value $v_{\sigma}=0.317$ is determined.

\section{DETERMINATION OF THE STRESS STATE OF THE CB}

The object of research in this work is the CB of a seven-piston APHM 210.25 (Fig. 3). The quantity of cylinders in the injection zone is variable, 3 or 4 , respectively in the suction zone -4 or 3 .

The large volumes of axial cavities being loaded and the variability of pressure along their length cause a complex, cyclically variable nature of the stress fields that in the $\mathrm{CB}$ arise.
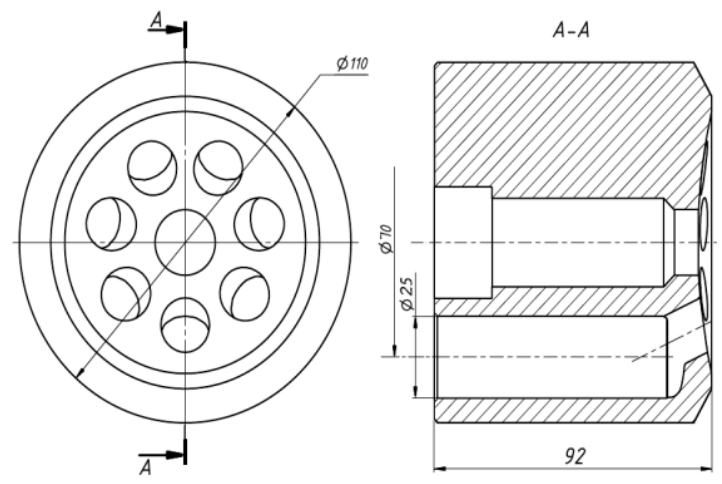

Fig. 3. The cylinder block of a hydraulic machine 210.25

Known models for the analytical calculation of the $\mathrm{CB}$ are either too simplistic (the cylinder was considered as a pipe of constant thickness, loaded with internal pressure) or bulky. Therefore, for stress field calculations FEM is used. These calculations at a pressure $p=25 \mathrm{MPa}$ for a various quantity of axial cylindrical holes in the injection zone were performed. It is established that the greatest magnitude of stresses is achieved when four axial channels are located in the injection zone. Some results of the FEM-calculation are shown in Fig. 4. It is installed that the maximum values of the first principal stress of $\sigma_{\max }=102 \div 104 \mathrm{MPa}$ act in the ICP on a section about $3 \mathrm{~mm}$ long. The region of action of stresses with a level of $94 \div 104 \mathrm{MPa}$ has a length of about $22 \mathrm{~mm}$, Fig. $4 a$.

It should be borne in mind that it is not possible to obtain exact values of stresses in many cases. Therefore, the recommendation of the standard that as $L$ should be assigned as a "part of the perimeter of the working section, which is adjacent to the zone of increased tension" in this case does not allow unambiguous determination of the value of this parameter. Below we propose a calculation method for determining the length $L$, based on the positions of the STSFF.

During APHM operation, stresses in the CB are changed by a pulsating cycle, whereas the STSFF methodology establishes procedures for calculating the fatigue resistance characteristics for the symmetrical stress cycle. Therefore, for further calculations, the resulting stress values are reduced to an equivalent symmetrical cycle.

Calculations are performed according to the Soderberg's formula:

$$
\sigma_{-1 e}(x, z)=\sigma_{0.2} \cdot \sigma_{0}(x, z) /\left[2 \sigma_{0.2}-\sigma_{0}(x, z)\right] .
$$


The stresses shown in Fig. $4 c$ and recalculated according to expression (8) are described by approximating functions:

$$
\begin{gathered}
\sigma_{-1 e}(x)=73-15.6 x+2.7 x^{2}, \\
\sigma_{-1 e}(z)=55+18 \cos [\pi(z+0.5) / 28] .
\end{gathered}
$$

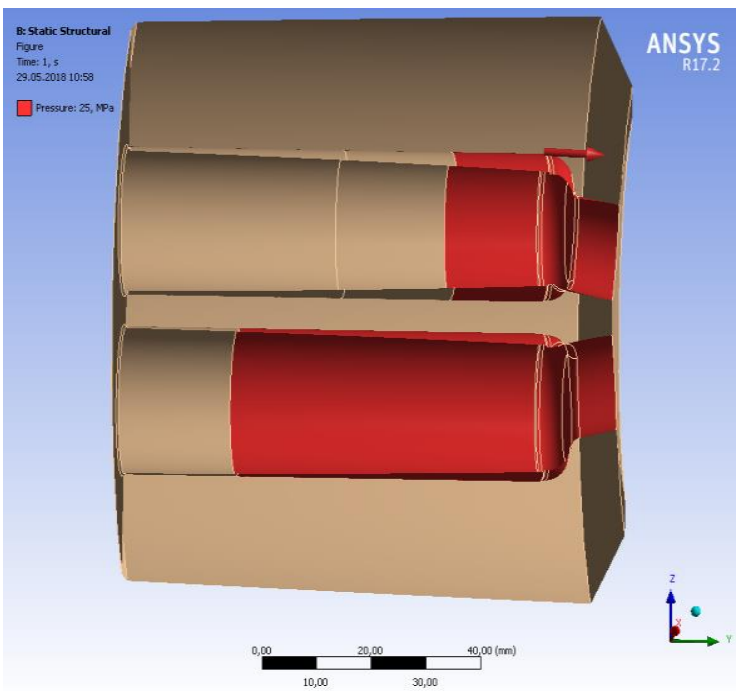

a) Pressure distribution $p=25 \mathrm{MPa}$ along the length of the cylinders

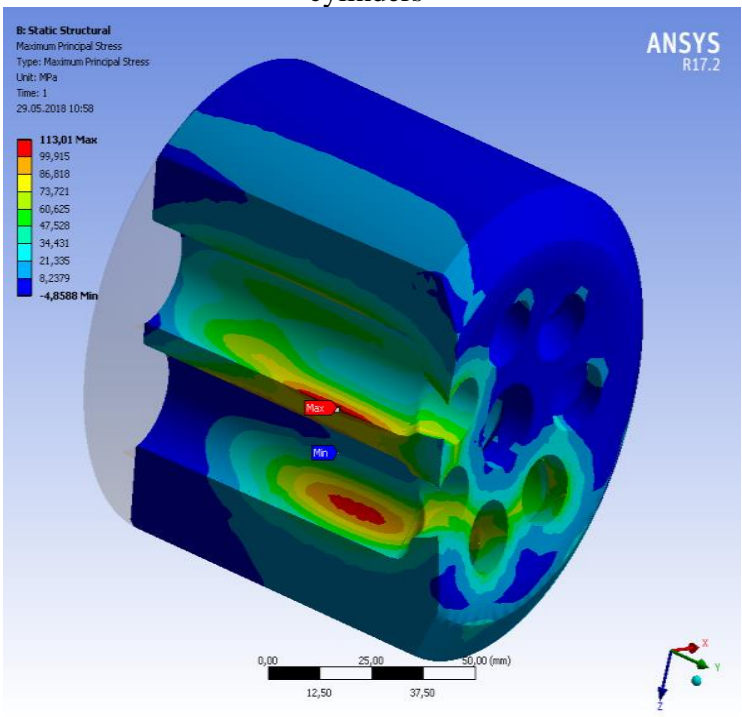

b) Region of localization of dangerous stresses

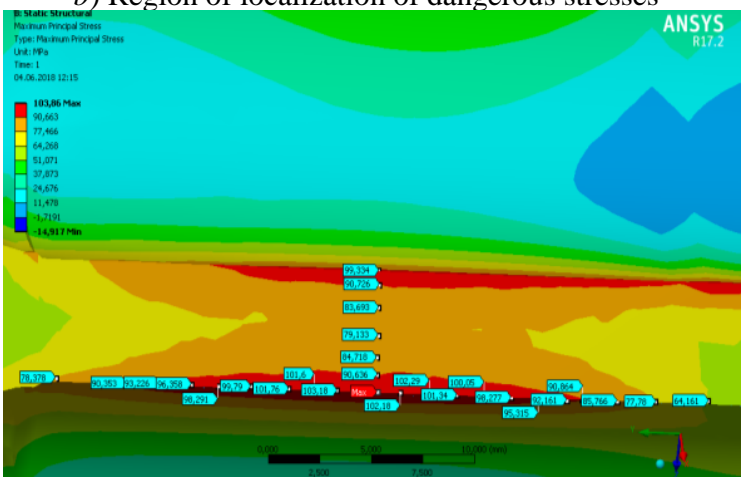

c) Distribution of the first main stress in the cross section of the ICP at its localization region

Fig. 4. The stressed state of the CB of APHM 210.25 in the vicinity of $\sigma_{0 \max }$
The stress diagrams (9) and (10) are shown in Fig. 5. The dots represent the stresses recalculated from formula (8).

Stresses, the value of which is below the boundary values $u=55 \mathrm{MPa}$, do not lead to the accumulation of fatigue damages and into calculations are not taken.

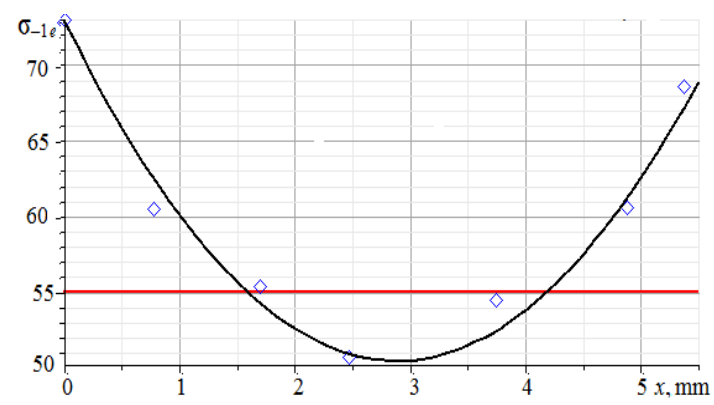

a) In the circumferential direction

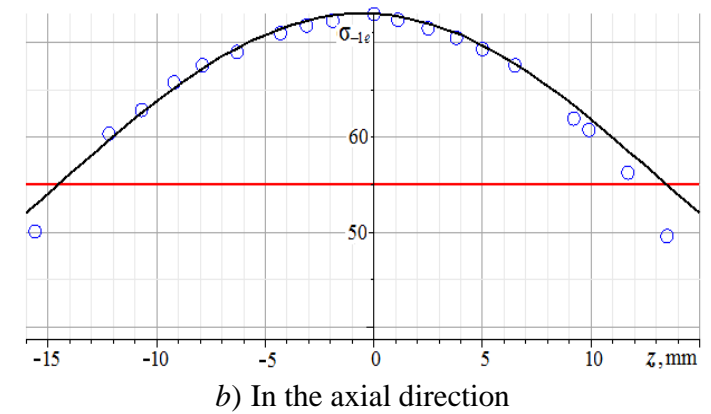

Fig. 5. Diagrams of equivalent stresses in the ICP:

The TSCF in the ICP is determined using the dependence (9)

$$
K_{t}=t_{0} \cdot \sigma_{-1 e}(0) / \int_{0}^{t_{0}} \sigma_{-1 e}(x) d x=1.28,
$$

where $\sigma_{-1 e}(0)=\sigma_{\max }=73 \mathrm{MPa}-$ the maximum equivalent stress. The ICP thickness is (Fig. 3)

$$
t_{0}=D_{0} \sin \frac{\pi}{7}-d_{0}=70 \sin \frac{\pi}{7}-25=5.37 \mathrm{~mm} \text {. }
$$

For the cross-sectional of the ICP the equation of surface of the equivalent stresses is obtained by combining expressions (9) and (10)

$$
\sigma_{-1 e}(x, z)=55+2.7 x^{2}-15.6 x+18 \cos \frac{\pi(z+0.5)}{28}
$$

The surface of equivalent stresses (11) is shown in Fig. 6.

The stresses in the longitudinal section of the partition $\sigma_{-1 e}(x, z)$ accept the maximum values at $x=0$.

When calculating the relative stress gradient $\bar{G}$, from these values we should take the largest, $\sigma_{\max }=73 \mathrm{MPa}$. As a result, is obtained

$$
\bar{G}=\frac{1}{\sigma_{\max }} \cdot\left|\frac{d \sigma_{-1 e}(x, 0)}{d x}\right|_{x=0}=0.214 \mathrm{~mm}^{-1}
$$




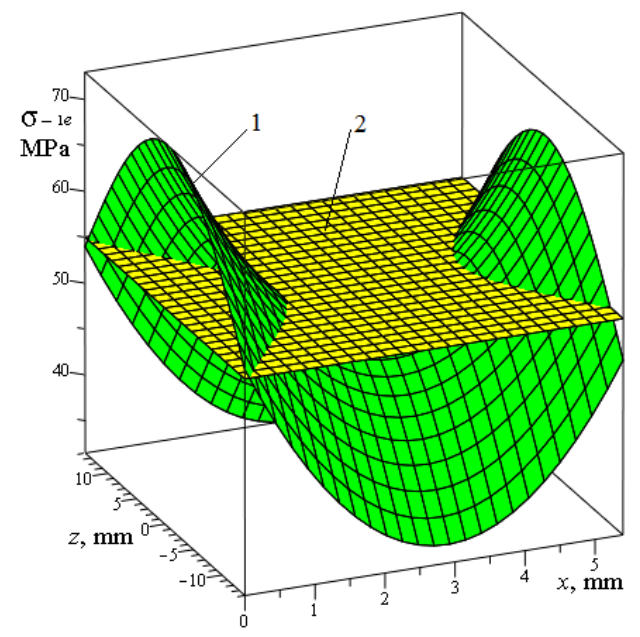

Fig. 6. The stress distribution model for cross-sectional of the ICP: $1-\sigma_{-1 e}(x, z)$ by the equation (11); 2 - the boundary plane $u=55 \mathrm{MPa}$.

\section{DETERMINATION OF THE EQUIVALENT LENGTH $L_{e}$ OF THE DANGEROUS ZONE}

For $\mathrm{CB}$ and similar details in which the stresses are distributed unevenly the value of $L$, determined by the recommendations of [16], may be unreasonably low, down to the level zero. To solve such problems, we propose a method of replacing the parameter $L$ by its equivalent of $L_{e}$. The equivalence criterion is the coincidence of the distribution functions of the limits of endurance of a real detail (CB) and her analogue, which is characterized by the constancy $\sigma_{\max }$ on the length of $L_{e}$. In accordance with the STSFF, the equivalence condition is determined by the similarity equation in the form [22]:

$$
\begin{aligned}
& I_{1}=\frac{4}{F_{0} \sigma_{W}^{\omega}} \int_{0}^{1.59} d x \int_{-g(x)-0.5}^{g(x)-0.5}\left[\sigma_{-1 e}(x, z)-u\right]^{\omega} d z= \\
& =\frac{L_{1 e}}{\bar{G} F_{0}}\left(\frac{u}{\sigma_{W}}\right)^{\omega} \cdot \frac{(\xi-1)^{\omega+1}}{\xi(\omega+1)}
\end{aligned}
$$

where $u, \omega$ and $\sigma_{W}$ - the Weibull's distribution parameters; $\xi=\sigma_{\max } / u ; F_{0}-$ an arbitrary quantity having the dimension of the area. The formula [21] $\omega_{1,2}=0.62 /\left(S_{y 1,2}-1\right)$ was used to determine the Weibull's distribution parameter $\omega$. The average value $\omega=2.51$ is obtained. In the problem under consideration, the parameter $\xi=73 / 55=1.33$.

In the integral (12) the limits of integration are determined by the equation of the line of intersection of the plane $u=55 \mathrm{MPa}$ with the surface $\sigma_{-1 e}(x, z)$, Fig. 6 . The function $g(x)$ in equation (12) is obtained in the form of

$$
g(x)=\frac{28}{\pi} \arccos \left(\frac{15.6 x-2.7 x^{2}}{18}\right) .
$$

Equation (12) is solved with relation to the length $L_{1 e}$. With the $\omega=2.51$ found above and the calculated value of the double integral in the formula (12), $I_{2}=5645$ for is $\xi=\sigma_{\max } / u=1.33$,

$$
L_{1 e}=4 \xi(\omega+1) u^{-\omega}(\xi-1)^{-1-\omega} I_{2} \bar{G}=12.5 \mathrm{~mm} .
$$

When calculating the equivalent length according to the rules of STSFF, it is necessary to sum the lengths of all equally hazardous areas. The localization of dangerous tensions after the transition of a rotating CB through an upper dead center arises too on the reverse surface of this ICP, see Figures $4 c$ and 6. This situation will take place for all partitions. Consequently, the value of the calculated equivalent length will be $L_{e}=2.7 \quad L_{1 e}=175 \mathrm{~mm}$ for the CB of seven-piston APHM.

\section{THE ENDURANCE LIMIT CALCULATION BY THE SIMILARITY EQUATION}

For the $\mathrm{CB}$ was obtained the value of the parameter

$$
\theta=\left(L_{e} / \bar{G}\right) /\left(L_{0} / \bar{G}_{0}\right)=9.24 .
$$

The values of the remaining parameters of equation (1) are found. The parameter $K_{F}$, which takes into account the surface roughness, at $R z=1.6 \mu \mathrm{m}$ is determined by the formula [16]

$$
K_{F}=1-0.22 \cdot\left(\log \frac{\sigma_{u}}{20}-1\right) \cdot \log R_{Z}=0.99 .
$$

The coefficient of anisotropy (at $\sigma_{u}<600 \mathrm{MPa}$ ), $K_{A}=0.9$. The hardening coefficient $K_{V}=1$ (no hardening) [16].

According to formula (1), $\bar{\sigma}_{-1 D}=57.4 \mathrm{MPa}$ is calculated. During operation of the APHM the stresses in the $\mathrm{CB}$ vary in the pulsating cycle from $\sigma=0$ until $\sigma_{\max }=\bar{\sigma}_{0 D}$. The mean and amplitude stresses in this case are the same, $\sigma_{m}=\sigma_{a}=0,5 \sigma_{\max }=0,5 \bar{\sigma}_{0 D}$. The value of the endurance limit for a pulsating cycle is recalculated according to the found value $\bar{\sigma}_{-1 D}$ from the Soderberg's formula $\sigma_{a} / \bar{\sigma}_{-1 D}+\sigma_{m} / \sigma_{0.2}=1$ in the view $\bar{\sigma}_{0 D}=2 \bar{\sigma}_{-1 D} \cdot \sigma_{0.2} /\left(\bar{\sigma}_{-1 D}+\sigma_{0.2}\right)$. Calculation gives $\bar{\sigma}_{0 D}=87.0 \mathrm{MPa}$. Resource tests of a group of hydro machines 210.25 found that the endurance limit of the $\mathrm{CB}$ corresponds to a pressure of 18.2 MPa [23]. For CB seven-piston APHM the maximum stress in her ICP is defined as a linear function of pressure, $\sigma=5.16 p$ [24]. Consequently, the experimentally established endurance limit of the $\mathrm{CB}$ is $\bar{\sigma}_{0}=93.9 \mathrm{MPa}$. Consequently, the calculated endurance limit is less than the experimentally established value by $7.9 \%$.

The error estimation of the calculated value of the endurance limit $\bar{\sigma}_{-1 D}$ was performed for the probability of non-destruction of $P=0.9$ (90\%), according to the standard method [16], which we used in [25]. The coefficient of variation of this quantity is determined taking into account the errors of individual parameters 


$$
v_{\sigma_{-1 D}}=\sqrt{v_{\sigma_{\max }}^{2}+v_{\sigma_{-1}}^{2}+v_{K_{t}}^{2}} .
$$

The calculations of stress $\sigma_{\max }$ using the FEM were performed. The finite elements of the optimal form, ten-node tetrahedrons were used. Their number was increased to 10723 units, which for stress $\sigma_{\max }$ the relative error $\varepsilon_{\sigma}=0.03$ is gives. The coefficient of variation of maximum stresses by the formula $v_{\sigma_{\max }}=\varepsilon_{\sigma} / z_{P}$ was found. The quantile of the normal distribution for a given probability $z_{0.9}=1.28$, therefore, $v_{\sigma_{\max }}=0.03 / 1.28=0.0234$.

The coefficient of variation of the $\mathrm{CB}$ material endurance limit has a range of $0.04-0.10$ [16]. In the calculation takes the average value $v_{\sigma_{-1}}=0.07$.

The coefficient of variation for $K_{t}$, as a function of the curvature radius $\rho_{0}$ of the stress concentration zone, is determined by the formula [16]

$$
v_{K t}=\left|\frac{\partial K_{t}}{\partial \rho_{0}}\right|_{0} \cdot \frac{\bar{\rho}_{0}}{\bar{K}_{t}} \cdot v_{\rho},
$$

where $\bar{K}_{t}$ and $\bar{\rho}_{0}$ are, respectively, the average values of TCSF and the radius of curvature of the stress concentration zone (i.e. in a dangerous cross section); $v_{\rho}=S_{\rho} / \bar{\rho}_{0}-$ coefficient of variation of the radius of curvature, $S_{\rho}$ - mean square deviation of the radius $\bar{\rho}_{0}$.

The function $K_{t}\left(\rho_{0}\right)$ is determined by the geometry of the detail in the stress concentration zone. In the considered problem, this is the zone of the minimum thickness of the jumper, Fig. $4 c$, which is presented as a plate with bilateral cuts at tension. For the $K_{t}\left(\rho_{0}\right)$ formula (3) with parameters $K_{t 1}$ and $K_{t 2}$ is valid. The considered ICP has a notch depth $t=\rho_{0}=0.5 d_{0}$ and by the formula (4) $K_{t 1}=3$ obtained. For the second parameter, the formula from [16] is used

$$
K_{t 2}=\frac{2\left(1+\zeta_{1}\right) \sqrt{\zeta_{1}}}{\sqrt{\zeta_{1}}+\left(1+\zeta_{1}\right) \cdot \operatorname{arctg} \sqrt{\zeta_{1}}}
$$

where $\zeta_{1}=0.5 t_{0} / \rho_{0}$

The calculation of the derivative in equation (14) for axial hole with diameter $25 \mathrm{H} 7\left(\begin{array}{l}+0.021 \\ 0\end{array}\right) \mathrm{mm}$, i.e. at $\bar{\rho}_{0}=12.5 \mathrm{~mm}$ gives the value of $\left|\frac{\partial K_{t}}{\partial \rho_{0}}\right|_{0}=0.0104$. The mean square deviation of the radius is taken to be equal to half the tolerance of the diameter of the hole, i.e. $S_{\rho}=0.01 \mathrm{~mm}$.

At these values, according to formula (14), a quantity $v_{K t}=8.5 \cdot 10^{-5}$ is obtained that can be neglected.
Calculation by the formula (13) of the coefficient of variation of the endurance limit of a detail gives the value $v_{\sigma_{-1 D}}=0.0738$.

Analysis of coefficient of variation $v_{\sigma_{-1 D}}$ shows that its value depends to the greatest degree on the accuracy of estimate. $\bar{\sigma}_{-1}$. An increase in the accuracy of this estimate and a corresponding decrease in the coefficient of variation $v_{\sigma_{-1}}$ require a significant rising in the number of tests of samples and is impractical. The value $v_{\sigma_{\max }}=0.0234$ is relatively small, moreover, due to the currently achieved high accuracy of the FEM, the determination error can be reduced to $\varepsilon_{\sigma}=0.005$, i.e. $0.5 \%$. At the accuracy, typical for the manufacture of hydraulic machine details, the coefficient of variation $v_{K t}$ is negligible.

It follows from the structure of formula (13) that the coefficients of variation for the symmetric and pulsating cycles will be the same, $v_{\sigma_{0 D}}=v_{\sigma_{-1 D}}$. The calculation of the endurance limit with a probability of $P=90 \%$ is performed according to the formula $\sigma_{0 D}=\bar{\sigma}_{0 D} \cdot\left(1 \pm z_{0.9} \cdot v_{\sigma_{0 D}}\right)$. The range of the calculated endurance limit at the indicated probability is $\sigma_{0 D}=(78.8 \div 95.3) \mathrm{MPa}$.

Therefore, the experimentally established value of $\sigma_{0}=93,9 \mathrm{MPa}$ is in this range. Thus, the developed calculation estimate of the value $\sigma_{0}=93,9 \mathrm{MPa}$ can be recommended for designing a wide class of complex shape details with an inhomogeneous distribution of peak stresses. This technique can be useful in finding the optimal structural forms of new details.

\section{DISCUSSION OF THE RESEARCH RESULT}

Existing normative documents do not contain recommendations for calculating the characteristics of fatigue resistance of complex shape parts in which significant local stresses arise. Here is a method for estimating the endurance limit of this type of details, confirmed by testing the CB APHM resource. This method should be regarded as an extension of the STSFF provisions to details with a localized stress distribution. The fatigue tests of smooth and notched samples from the CB material made it possible to construct fatigue curves for bronze CuSn12 and determine the parameters of the similarity equation $u, \omega$ and $v_{\sigma}$.

A necessary additional element of the procedure is the finite element analysis of the stressed state of the detail under investigation, on the basis of which are defined the gradient of the first principal stress, the stress concentration coefficient and the equivalent analog of the parameter $L$. This made it possible effectively the fatigue fracture similarity 
equation to use for calculating the endurance limit of a complex shaped detail, in this case - the CB.

While continuing the development of the proposed method, it is advisable to consider the possibility of using it for calculating parts with a complex stress distribution for arbitrary cycle asymmetry and irregular loading.

\section{CONCLUSIONS}

1. Tests of material were performed, fatigue curves were plotted and the main parameters of the fatigue fracture similarity equation, necessary for calculating the endurance limit of the $\mathrm{CB}$ of APHM, were established.

2. With the use of FEM, the nature of the stressed state of the CB of APHM was established, the analysis of which made it possible to determine the area of possible destruction of the part and calculate the characteristics of stress concentration in this region.

3. A numerical and analytical method based on the statistical theory of the similarity of the fatigue fracture of Serensen-Kogayev has been developed, which made it possible to determine the endurance limit of the CB of APHM if a limited volume of sample testing. The acceptability of the proposed calculation technique for estimating the endurance limit of parts with the localization of operating stresses is certified on the results of full-scale tests.

4. The error estimation of the proposed theoreticalnumerical method based on the calculation of the coefficient of variation of the endurance limit is performed. In the considered example, it was shown that the error of the method used does not exceed $7.9 \%$.

\section{REFERENCES}

1. Kutuzov VK, Voronov SA, Sergeev YV. Investigation of the influence of loading regimes of axial-piston (in Russian). Hydraulic Machines on the Duration of their Tests. Assembling in Mechanical Engineering. Instrument-Making.2015; 95(6): 38-41.

2. Larchikov I, Yurov A, Stazhkov S et al. Analysis of an axial piston hydraulic machine of power intensive hydraulic drive system. Procedia Engineering. 2014; 69: 512-517. https://doi.org/10.1016/j.proeng.2014.03.020

3. Puzanov AV. Experience in using modern multiphysical software in the development of electrohydraulic actuators. (in Russian). CAD and Graphics.2008; 4: 75-79.

4. Schuhler G, Jourani A, Bouvier S, Perrochat JM. Multi Technical Analysis of Wear Mechanisms in Axial Piston Pumps. Journal of Physics.2017; Conf. Ser. 843 012077: 15 p.

5. Avrunin GA, Samorodov VB, Moroz II. The study of power losses in axial piston hydraulic machines (in Russian). Bulletin of National Technical University "KhPI".2017; 1264(42): 56-59.

http://repository.kpi.kharkov.ua/handle/KhPIPress/33695
6. Bergada JM, Kumar S, Davies DL, Watton J. A complete analysis of axial piston pump leakage and output flow ripples. Applied Mathematical Modelling.2012; 36 (4): 1731-1751.

7. Ebara R. Some influencing variables on internal fatigue crack initiation in structural materials. Procedia Engineering.2016; 160: 21 - 28. https://doi.org/10.1016/j.proeng.2016.08.858

8. Erny C, Thevenet D, Cognard JY, Körner M. Fatigue assessment of naval welded assemblies. Procedia Engineering.2010; 2: 603-612.

9. Van Wittenberghe J, De Pauw J, De Baets P. Experimental determination of the fatigue life of modified threaded pipe couplings. Procedia Engineering.2010; 2 (1): 1849-1858.

10. Sandu N, Zaharia NL. Static and dynamic tests performed on a flat wagon. Problemy Kolejnictwa. 2014; 163: 67-77.

11. Vira V, Kulyk V, Chepil R, Kharchenko Y, Duriagina $Z$. The diagnostics and ways heat treatment optimization of a railway wheels steel. Diagnostyka. 2019;20(2):105-111. https://doi.org/10.29354/diag/109605

12. Kazuhiko Kasai, Hiroshi Ito, Shojiro Motoyui et al. Full-Scale shake table tests of 5-story steel building with various dampers. Joint conference proceedings 7th International Conference on Urban Earthquake Engineering (7CUEE) \& 5th International Conference on Earthquake Engineering (5ICEE). 2010; 11-22.

13. Będkowski W. Assessment of the fatigue life of machine components under service loading - a review of selected problems. Journal of Theoretical and Applied Mechanics, Warsaw.2014; 52 (2): 443458.

14. Wang H, Meng FL. Simulation and optimal design of cylinder block of axial piston pump based on ANSYS, J. Advan. Mat. Res.2011; 186: 368-372. https://doi.org/10.4028/www.scientific.net/AMR.186. 368

15. DIN 743-2:2012-12. Tragfähigkeitsberechnung von Wellen und Achsen.- Teil 2: Formzahlen und Kerbwirkungszahlen (in German)

16. GOST 25.504-82 Calculations and strength tests. methods for calculating fatigue resistance characteristics (in Russian).

17. Stepnov MN. Calculational methods for constructing curves of multi-cyclic fatigue taking into account structural factors (in Russian). Bulletin of Machine Building.2012; 9: 17-24.

18. Cera A, Mancini G, Leonardi V, Bertini L. Analysis of methodologies for fatigue calculation for railway bogie frames. 8th World Congress on Railway Research R.1.1.3.2, Seoul. 2008; 13 p.

19. Zheglova V, Khomyak Y, Medvedev S, Nikolenko I. Numerical and analytical evaluation of service life of the details axial piston hydraulic machines with complicated configuration under cyclic loading. Procedia Engineering.2017; 176: 557-566.

20. Quan Ling-Xiao, Cao Yuan, Luo Hong-liang, Guo Rui, Guo Haixin. Fatigue analysis of the cylinder in the axial piston pump. Conference Paper. August 2015: 1-8. https://doi. 10.1109/FPM.2015.7337096

21. Oleynik NV, Medvedev SA. The method of rectifying the hyperbolic profile of the groove on fatigue specimens (in Russian). Machine Elements: The Republican Interdepartmental Scientific and Technical Proceedings.1974; 18: 99-105. 
22. Kogaev VP, Makhutov NA, Gusenkov AP. Calculations of machine elements and structures for strength and durability: Handbook (in Russian). Moscow, Mashinostroyeniye Publ.1985; 224.

23. Dodin LG. Methods of testing axial-piston hydraulic machines (in Russian). Proceedings of VNIIStroydormash. 1981; 92: 23-29.

24. Nikolenko IV, Khomyak YM, Kibakov AG. Calculation of the durability the cylinder block of hydraulic machines (in Russian). Bulletin of Machine Building. 1988; 2: 26-29.

25. Gutyrya SS, Medvedev SA, Khomiak YM, Chanchin AV. Probabilistic analysis of fatigue durability of an epicycle of a wheel gearbox of the trolleybus. Bulletin of NTU "KhPI". Series: Problem of mechanical drive. -Kharkiv: NTU "KhPI", 2017; 25(1247):37-43.

\section{Received 2019-07-08}

Accepted 2020-01-20

Available online 2020-01-21
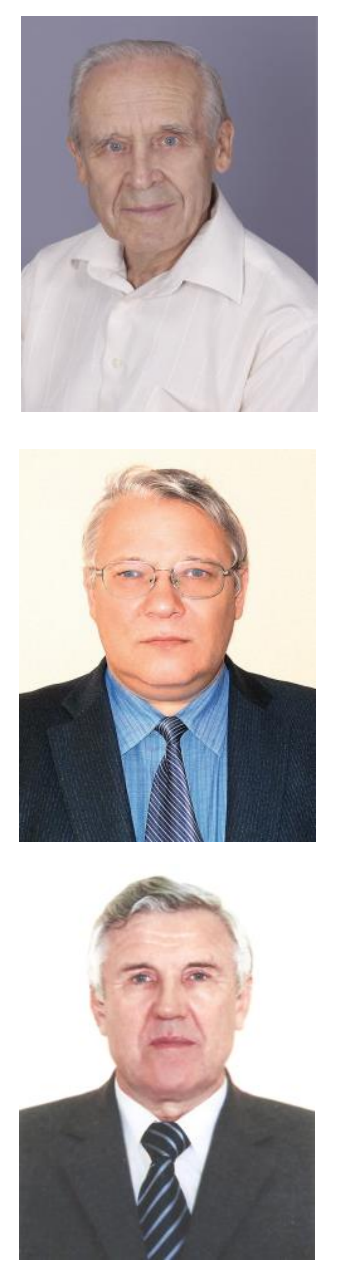

Oleksandr KIBAKOV has received his $\mathrm{PhD}$ in Technical Sciences in 1994. Assistant professor, currently he is Dean of the Faculty of Engineering of the Port of Odessa National Maritime University.

Stanislav MEDVEDEV has received his $\mathrm{PhD}$ in Technical Sciences in 1981. Currently he is assistant professor of "Machine Science" Department in Odessa National Maritime University.

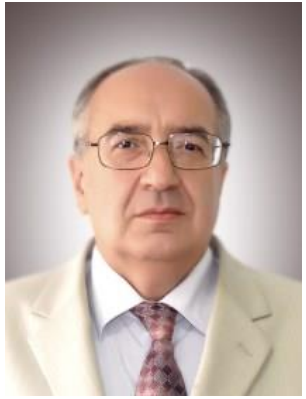

Ilya NIKOLENKO has received his Dr.Sn in Technical Sciences in 2006.Professor, currently he is Head of Department "Water supply, sanitation and sanitary equipment" in Academy of Construction and Architecture "V.I. Vernadsky Crimean Federal University".

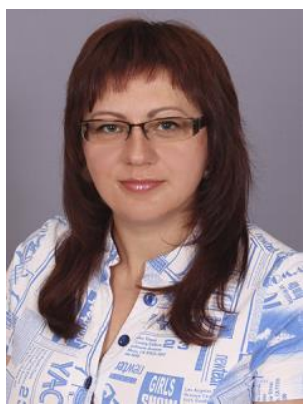

Victoria ZHEGLOVA has received his $\mathrm{PhD}$ in Technical Sciences in 2015. Currently he is assistant professor of Department of metal-cutting machine tools, metrology and certification in Odessa National Polytechnic University. 